\title{
Thousand per High Powered Field
}

National Cancer Institute

\section{Source}

National Cancer Institute. Thousand per High Powered Field. NCI Thesaurus. Code C98789.

A unit of measurement equal to one thousand entities per unit of area equal to one high powered field. 